Pacific Journal of Mathematics 


\section{A CLASS OF RESIDUE SYSTEMS $(\bmod r)$ AND RELATED ARITHMETICAL FUNCTIONS, I. A GENERALIZATION OF MÖBIUS INVERSION}

\section{ECKFORD COHEN}

1. Introduction. Let $Z$ denote the set of positive integers and let $P$ and $Q$ be nonvacuous subsets of $Z$ such that if $n_{1} \in Z, n_{2} \in Z$, $\left(n_{1}, n_{2}\right)=1$, then

$$
n=n_{1} n_{2} \in P \rightleftarrows n_{1} \in P, n_{2} \in P ;
$$

suppose also that the elements $n$ in $Q$ satisfy the condition (1.1) with $P$ replaced by $Q$. If, in addition, every integer $n \in Z$ possesses a unique factorization of the form

$$
n=a b, \quad a \in P, b \in Q,
$$

then each of the sets $P$ and $Q$ will be called a direct factor set of $Z$, while $P$ and $Q$ together will be said to form a conjugate pair. In the rest of this paper $P$ will denote such a direct factor set with conjugate set $Q$. It is clear that 1 is the only integer common to both $P$ and $Q$. A simple example of a conjugate pair $P, Q$ is the set $P$ consisting of 1 alone and the set $Q=Z$.

Let $r$ be a positive integer. In this paper we shall generalize the notion of a reduced residue system $(\bmod r)$. If $P$ is a given direct factor set, then the elements $a$ of a complete residue system $(\bmod r)$ such that $(a, r) \in P$ will be called a $P$-reduced residue system $(\bmod r)$ or simply a $P$-system $(\bmod r)$. Any two $P$-system $(\bmod r)$ are equivalent in the sense that they are determined by the residue classes of the integers $(\bmod r)$. A $P$-system chosen from the numbers $1 \leq a \leq r$ will be called a minimal $P$-system $(\bmod r)$. The number of elements in a $P$-system $(\bmod r)$ will be denoted by $\phi_{P}(r)$ and called the $P$-totient of $r$. Clearly, if $P=1, \phi_{P}(r)$ reduces to the ordinary Eulerian totient $\phi_{1}(r)=\phi(r)$, while $\phi_{Z}(r)=r$.

We summarize here the central points of the paper. Analogous to the generalization $\phi_{P}(r)$ of $\phi(r)$, we define in $\S 2$ a function $\mu_{P}(r)$ extending the Möbius function $\mu(r)$ to arbitrary direct factor sets $P$. On the basis of this definition we prove in Theorem 3 an analogue of the Möbius inversion formula. This result is then applied in $\S 3$ to yield an evaluation of $\phi_{P}(r)$. In $\S 4$ a generalization $c_{P}(n, r)$ of Ramanujan's trigonometric sum $c(n, r)$ is defined and evaluated for arbitrary direct factor sets.

Received August 26, 1958. 
In $\$ 5$ applications to two relative partition problems $(\bmod r)$ are considered. In particular, in Theorem 12 we obtain a formula for the number of solutions $(\bmod r)$ of the congruence

$$
n \equiv x_{1}+\cdots+x_{s} \quad(\bmod r),
$$

such that $\left(x_{i}, r\right) \in P,(i=1, \cdots, s)$. In Theorem 13 a formula is deduced for the number $\theta_{P}(n, r)$ of integers a $(\bmod r)$ such that $(a, r)=1$ and $(n-a, r) \in P$. These two theorems are wide generalizations of results proved by the author in [1], [2], and [3]. We remark that the method in $\S 5$ and the latter part of $\S 4$ is based on the theory of even functions $(\bmod r)$ developed in the three papers cited above.

In $\S 6$ the results of the preceding sections are specialized to the conjugate pair $P, Q$, where $P$ consists of the $k$-free integers and $Q$ is the set of $k$ th powers. Precise criteria for the vanishing of $\theta_{P}(n, r)$ and $\theta_{Q}(n, r)$ in these cases will be found in Theorem 14 .

Regarding the theoretical foundations of arithmetical inversion, we mention an investigation of Hölder [6]. Additional references to the literature appear in Hölder's paper.

REMARK. It is noted that several of the results proved in this paper are valid for arbitrary sets $P$, as distinguished from direct factor sets (for example, Theorems 6, 8, 9, and 13). In the general case, however, the unifying method of arithmetical inversion is no longer applicable. The broader topic of arthmetical functions in relation to arbitrary sets $P$ will be treated in another paper.

2. The inversion function $\mu_{p}(r)$. We recall the following fundamental property of $\mu(r)$.

$$
\sum_{a \mid r} \mu(d)=\rho(r) \equiv\left\{\begin{array}{ll}
1 & (r=1) \\
0 & (r>1)
\end{array} .\right.
$$

The $\mu$-function may be generalized to arbitrary direct factor sets by writing

$$
\mu_{P}(r)=\sum_{\substack{d \backslash r \\ d \in P}} \mu\left(\frac{r}{d}\right)
$$

where the summation is over the divisors $d$ or $r$ contained in $P$. It will be observed that $\mu_{1}(r)=\mu(r)$ and $\mu_{Z}(r)=\rho(r)$.

By (2.2), (1.1), and the factorability of $\mu(r)$, it follows that $\mu_{P}(r)$ is a factorable function of $r$ :

THeOREM 1. If $r_{1} \in J, r_{2} \in J,\left(r_{1}, r_{2}\right)=1$, then 


$$
\mu_{P}(r)=\mu_{P}\left(r_{1}\right) \mu_{P}\left(r_{2}\right), \quad\left(r=r_{1} r_{2}\right) .
$$

We next prove that the property (2.1) of $\mu(r)$ can be extended to the function $\mu_{P}(r)$.

\section{THEOREM 2.}

$$
\sum_{\substack{d \mid r \\ d \in Q}} \mu_{P}\left(\frac{r}{d}\right)=\rho(r)
$$

Proof. On the basis of (2.1), (2.2) and the uniqueness of the factorlzation (1.2) one obtains

$$
\begin{aligned}
\sum_{\substack{d \mid r \\
a \in Q}} \mu_{P}\left(\begin{array}{c}
r \\
d
\end{array}\right) & =\sum_{\substack{d=r=r \\
a \in Q}} \sum_{\substack{\rho=\delta D \\
\delta \in P}} \mu(D) \\
& =\sum_{D \mid r} \mu(D) \sum_{\substack{\delta a=r \mid D \\
\delta \in P, d \in Q}} 1=\sum_{D \mid r} \mu(D)=\rho(r) .
\end{aligned}
$$

This completes the proof.

By means of Theorem 2 we generalize the Möbius inversion formula to arbitrary direct factor sets.

THEOREM 3. If $f(r)$ and $g(r)$ are arithmetical functions, then

$$
f(r)=\sum_{\substack{d \mid r \\ a \in Q}} g\left(\frac{r}{d}\right) \rightleftarrows g(r)=\sum_{d \mid r} f(d) \mu_{P}\left(\frac{r}{d}\right) .
$$

Proof. Let $f(r)$ be defined as on the left of (2.5). By (2.4) one obtains

$$
\begin{aligned}
\sum_{d \mid r} f(d) \mu_{P}\left(\frac{r}{d}\right) & =\sum_{d \mid r}\left(\sum_{\substack{\delta \in=a \\
\delta \in Q}} g(e)\right) \mu_{P}\left(\begin{array}{c}
r \\
d
\end{array}\right) \\
& =\sum_{e \mid r} g(e) \sum_{\substack{d \delta^{\prime}=r \\
\delta=d \\
\delta \in Q}} \mu_{P}\left(\delta^{\prime}\right)=\sum_{e \mid r} g(e) \sum_{\substack{\delta \delta \\
\delta \in=r / e}} \mu_{P}\left(\delta^{\prime}\right) \\
& =\sum_{e \mid r} g(e) \rho\left(\frac{r}{e}\right)=g(r) .
\end{aligned}
$$

Conversely, let $g(r)$ be defined as on the right of (2.5). Then again by $(2.4)$

$$
\begin{aligned}
\sum_{\substack{d \mid r \\
a \in Q}} g\left(\begin{array}{c}
r \\
d
\end{array}\right) & =\sum_{\substack{d e=r \\
a \in Q}}\left(\sum_{\delta \mid e} f(\delta) \mu_{P}\left(\frac{e}{\delta}\right)\right) \\
& =\sum_{\delta \mid r} f(\delta) \sum_{\substack{d e=r \\
\delta \delta=0 \\
a \in Q}} \mu_{P}\left(\delta^{\prime}\right)=\sum_{\delta \mid r} f(\delta) \sum_{\substack{\alpha \delta^{\prime}=r / \delta \\
a \in Q}} \mu_{P}\left(\delta^{\prime}\right)
\end{aligned}
$$




$$
=\sum_{\delta \mid q} f(\delta) \rho\left(\frac{r}{\delta}\right)=f(r) \text {. }
$$

The proof is complete.

It is evident that if $P=1, Q=Z$, Theorem 3 becomes the inversion formula of elementary number theory.

3. The totient function $\phi_{P}(r)$. The following principle is basic in considering $P$-totients.

THEOREM 4. Let $d$ range over the divisiors of $r$ contained in $Q$, and for each such $d$ let $X$ range over the elements of a $P$-system (mod $r / d)$. Then the set $d X$ forms a complete residue system (mod $\mathrm{r}$ ).

Proof. In the proof we suppose $n$ to range over the positive integers $\leq r$. For a fixed divisor $d$ of $r, d \in Q$, let $C_{d}$ represent the set of those $n$ for which $(n, r)$ is of the form $(n, r)=d e, e \in P$. By the uniqueness of the factorization (1.2), a given $n$ lies in exactly one class $C_{d}$; hence the set of elements in the classes $C_{d}$ consists precisely of the integers $1, \cdots, r$. Moreover, for a fixed divisor $d$ of $r$ such that $d \in Q$, the elements $n=d x$ comprise $C_{a}$ if and only if $(x, r / d) \in P, 1 \leq x \leq r / d$, that is, if and only if the elements $x$ form a minimal $P$-system (mod $r / d)$. Replacing the particular $P$-system $x(\bmod r / d)$, by an arbitrary $P$-system $X(\bmod r / d)$ the theorem results.

Theorem 4 leads immediately to

Theorem 5.

$$
\sum_{\substack{d \mid r \\ d \in Q}} \phi_{P}\left(\frac{r}{d}\right)=r
$$

The evaluation of $\phi_{P}(r)$ follows from (3.1) on applying the inversion formula of Theorem 3 :

THEOREM 6.

$$
\phi_{P}(r)=\sum_{a \mid r} d \mu_{P}\left(\frac{r}{d}\right)
$$

In case $P=1$, Theorem 6 becomes the well-known evaluation formula for $\phi(r)$.

Since $\mu_{P}(r)$ is factorable (Theorem 1) the same is true of $\phi_{P}(r)$, by (3.2):

THEOREM 7. If $\left(r_{1}, r_{2}\right)=1$, then 


$$
\phi_{P}(r)=\phi_{P}\left(r_{1}\right) \phi_{P}\left(r_{2}\right), \quad\left(r=r_{1} r_{2}\right) \text {. }
$$

Next we show how $\phi_{P}(r)$ may be expressed in terms of the ordinary $\phi$-function.

THEOREM 8.

$$
\phi_{P}(r)=\sum_{\substack{d \mid r \\ d \in P}} \phi\left(\frac{r}{d}\right)
$$

Proof. By (2.2) and (3.2) it follows that

$$
\phi_{P}(r)=\sum_{\delta \mid r} \delta \sum_{\substack{d \mid r / \delta \\ d \in P}} \mu\left(\frac{r / \delta}{d}\right)=\sum_{\substack{d \mid r \\ d \in P}} \sum_{\delta \mid r / d} \delta \mu\left(\frac{r / d}{\delta}\right) .
$$

and (3.4) results by (3.2) with $P=1$.

4. The exponential sum $c_{P}(n, r)$. We define

$$
c_{P}(n, r)=\sum_{(x, r) \in P} e(x n, r), \quad e(a, r)=e^{2 \pi i a / r},
$$

where the summation is over a $P$-system $(\bmod r)$. In case $P=1, c_{P}(n, r)$ reduces to the Ramanujan sum, $c(n r)$. The next theorem generalizes the familiar evaluation of $c(n, r)$.

TheOREM 9.

$$
c_{P}(n, r)=\sum_{d \mid(n, r)} d \mu_{P}\left(\begin{array}{l}
r \\
d
\end{array}\right) .
$$

Proof. Placing $r(n, r)=c_{Z}(n, r)$, we have

$$
r(n, r)=\sum_{x(\bmod r)} e(x n, r)= \begin{cases}r & (r \mid n) \\ 0 & (r+n) .\end{cases}
$$

Furthermore, by Theorem 4,

$$
\eta(n, r)=\sum_{\substack{d \mid r \\ d \in Q}} \sum_{(x, r \mid a) \in P} e(d x n, r)=\sum_{\substack{d \mid r \\ d \in Q}} c_{P}\left(n, \frac{r}{d}\right) .
$$

Therefore, by the inversion theorem $(\S 2)$,

$$
c_{P}(n, r)=\sum_{d \mid r} \eta(n, d) \mu_{P}\left(\begin{array}{l}
r \\
d
\end{array}\right),
$$

and the theorem follows on the basis of (4.3).

The function $c_{P}(n, r)$ is a generalization of both $\phi_{P}(r)$ and $\mu_{P}(r)$ : 
COROLlaRY 9.1. If $n \equiv 0(\bmod r)$, then

$$
c_{P}(n, r)=\phi_{P}(r) \text {. }
$$

CoRollary 9.2 If $(n, r)=1$, then

$$
c_{P}(n, r)=\mu_{P}(r) .
$$

By (4.2) and (2.3) we have, in addition,

THEOREM 10. The function $c_{P}(n, r)$ is a factorable function of $r$; that is, if $\left(r_{1}, r_{2}\right)=1$, then

$$
c_{P}(n, r)=c_{P}\left(n, r_{1}\right) c_{P}\left(n, r_{2}\right), \quad\left(r=r_{1} r_{2}\right) .
$$

In the proof of the next theorem we assume the results on even functions $(\bmod r)$ proved in [1]. We first state a lemma which results on applying the Möbius-inversion formula to (2.2).

LEMMA 1.

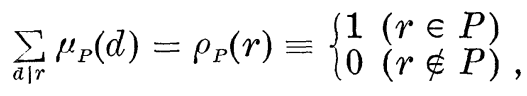

It is noted that $\rho_{1}(r)=\rho(r)$.

THEOREM 11.

$$
c_{P}(n, r)=\sum_{a \mid r} \rho_{P}\left(\frac{r}{d}\right) c(n, d)=\sum_{\substack{d \mid r \\ d \in P}} c\left(n, \frac{r}{d}\right) .
$$

Proof. By (4.2), $c_{P}(n, r)=c_{P}((n, r), r)$, so that $c_{P}(n, r)$ is an even function of $n(\bmod r)$. Hence by Theorem 9 and [1, Theorem 4], $c_{P}(n, r)$ has a Fourier expansion,

$$
c_{P}(n, r)=\sum_{a \mid r} \alpha(d, r) c(n, d),
$$

where

$$
\alpha(d, r)=\sum_{e \mid r / d} \mu_{P}(e),
$$

and the theorem follows by (4.8).

We note that (4.9) reduces to (3.4) in case $n=0$, thereby providing a new proof of Theorem 8 , while in case $n=1$, (4.9) becomes (2.2).

5. Relative partitions $(\bmod r)$. In this section we assume the results of [2] and [3]. Let $A_{p}^{(s)}(n, r)$ denote the number of solutions $(\bmod r)$ of (1.3), such that for each $x_{i},(1 \leq i \leq s),\left(x_{i}, r\right)$ is contained in a $P$-system 
$(\bmod r)$. We deduce the following expansion for $A_{p}^{(s)}(n, r)$.

THEOREM 12. For arbitrary positive integral $s$,

$$
A_{P}^{(s)}(n, r)=\frac{1}{r} \sum_{d \mid r}\left(c_{P}\left(\frac{r}{d}, r\right)\right)^{s} c(n, d) .
$$

Proof. We prove (5.1) inductively on $s$. Obviously $A_{P}^{(1)}(n, r)=$ $\rho_{P}((n, r))$. Hence applying [2, Theorem 3] to (4.9), one obtains

$$
A_{P}^{(1)}(n, r)=\frac{1}{r} \sum_{d \mid r} c_{P}\left(\frac{r}{d}, r\right) c(n, d) .
$$

This proves the theorem in case $s=1$. We assume the theorem for $s=t \geq 1$. Then by [3, Theorem 1]

$$
\begin{gathered}
A_{P}^{(t+1)}(n, r)=\sum_{n \equiv a+b(\text { inod } r)} A_{P}^{(t)}(a, r) A_{P}^{(1)}(b, r) \\
=\frac{1}{r} \sum_{a \mid r}\left(c_{P}\left(\begin{array}{l}
r \\
d
\end{array}, r\right)\right)^{t+1} c(n, d) .
\end{gathered}
$$

This completes the induction.

Next we derive an arithmetical formula for the function $\theta_{P}(n, r)$ defined in the Introduction. Equivalently $\theta_{P}(n, r)$ may be defined as the number of solutions, $x, y(\bmod r)$ of

$$
n \equiv x+y(\bmod r), \quad(x, r)=1, \quad(y, r) \in P .
$$

The proof will depend on the following lemma.

Lemma 2. Let $e$ be a positive integer. Then

$$
\sum_{a \mid r} c\left(\frac{r}{d}, e\right) \mu(d)= \begin{cases}\mu\left(\frac{e}{r}\right) r & \text { if } r \mid e, \\ 0 & \text { otherwise. }\end{cases}
$$

Proof. By the evaluation formula for $c(n, r)$,

$$
\begin{aligned}
\sum_{d \mid r} c\left(\frac{r}{d}, e\right) \mu(d) & =\sum_{d \mid r} \mu(d) \sum_{D \mid(r \mid a, e)} D \mu\left(\frac{e}{D}\right) \\
& =\sum_{D \mid(e, r)} \mu\left(\begin{array}{c}
e \\
D
\end{array}\right) D \sum_{a \mid r / D} \mu(d),
\end{aligned}
$$

and (5.4) follows on applying (2.1) to the inner sum of the last expression. 
THEOREM 13.

$$
\theta_{P}(n, r)=\phi(r) \sum_{\substack{d \mid r \\(d, n)=1}} \frac{\mu_{P}(d)}{\phi(d)}
$$

where the summation is over the divisors of $r$ prime to $n$.

Proof. Using (5.2) we apply [2 Theorem 6] to $\theta_{P}(n, r)$ with $f(n, r)=A_{p}^{(1)}(n, r)$, obtaining on the basis of Theorem 11 and Lemma 2,

$$
\begin{aligned}
& \phi^{-1}(r) \theta_{P}(n, r)=\frac{1}{r} \sum_{\substack{a \mid r \\
(a, n)=1}} d(d)\left(\sum_{\delta \delta^{\prime}=r / a} c_{P}\left(\delta^{\prime}, r\right) \mu(\delta)\right) \\
& =\frac{1}{r} \sum_{\substack{d \mid r \\
(\alpha, n)=1}} \frac{d}{\phi(d)} \sum_{\substack{e^{\prime} \in r \\
e^{\prime} \in P}}\left(\sum_{\delta \delta^{\prime}=r / a} c\left(\delta^{\prime}, e\right) \mu(\delta)\right) \\
& =\sum_{\substack{d \mid r \\
(a, n)=1}} \frac{1}{\phi(d)} \sum_{\substack{e e^{\prime}=r \\
(r) \\
e^{\prime} \in P=e}} \mu(E)=\sum_{\substack{a \mid r \\
(a, n)=1}} \frac{1}{\phi(d)} \sum_{\substack{E e^{\prime}=a \\
e^{\prime} \in P}} \mu(E),
\end{aligned}
$$

and the theorem follows by definition $\mu_{P}(r)$.

6. Special cases. For a fixed non-negative integer $k$, let $P$ be the set of all $k$-free numbers and let $Q$ be the set of all $k$ th powers. Clearly $P$ and $Q$ form a conjugate pair of direct factor sets. We introduce the following notation for the functions corresponding to these sets: $\Phi_{k}(r)=\phi_{P}(r), \mu_{k}(r)=\mu_{P}(r), g_{k}(n, r)=c_{P}(n, r)$, and $\Psi_{k}(r)=\phi_{Q}(r), \lambda_{k}(r)=$ $\mu_{Q}(r), h_{k}(n, r)=c_{Q}(n, r)$. If $(a, b)_{k}$ is defined to be the greatest $k$ th power divisor of $a$ and $b$, then $\Phi_{k}(r)$ denotes the number of integers $a$ $(\bmod r)$ such that $(a, r)_{k}=1$, while $\Psi_{k}(r)$ denotes the number of $a$ $(\bmod r)$ such that $(a, r)$ is a kth power, that is, $(a, r)_{k}=(a, r)$.

It is observed that, in case $k=1, \Phi_{k}(r), \mu_{k}(r)$, and $g_{k}(n, r)$ reduce to $\phi(r), \mu(r)$, and $c(n, r)$, respectively. We also note that $\lambda_{2}(r)=\lambda(r)$, where $\lambda(r)$ represents the Liouville function. The conjugate totient functions $\Phi_{k}(r)$, and $\Psi_{k}(r)$ were introduced by Rogel [9]. Regarding the special case $k=2$ of these two functions, $\Phi_{2}(r)$ was evaluated by Haviland [5] using a definition equivalent to that given here, while $\Psi_{2}(r)$ was evaluated by the author in [2, Corollary 4.2]. For a further discussion of the function $\Phi_{k}(r)$ we refer to McCarthy [7].

The following evaluation arise as corollaries of the results proved in $\S \S 3$ and 4 .

$$
\begin{aligned}
& \Phi_{k}(r)=\sum_{d \mid r} d \mu_{k}\left(\frac{r}{d}\right)=\sum_{\substack{d \mid r \\
(a, r)_{k}=1}} \phi\left(\frac{r}{d}\right), \\
& \Psi_{k}(r)=\sum_{d \mid r} d \lambda_{k}\left(\begin{array}{l}
r \\
d
\end{array}\right)=\sum_{d^{k} \mid r} \phi\left(\frac{r}{d^{k}}\right),
\end{aligned}
$$




$$
\begin{aligned}
& g_{k}(n, r)=\sum_{a \mid(n, r)} d \mu_{k}\left(\frac{r}{d}\right)=\sum_{\substack{d|r| r \\
(a, r)_{k}=1}} c\left(n, \frac{r}{d}\right), \\
& h_{k}(n, r)=\sum_{a \mid(n, r)} d \lambda_{k}\left(\frac{r}{d}\right)=\sum_{a^{k} \mid r} c\left(n, \frac{r}{d} k\right) .
\end{aligned}
$$

By (2.2) the functions $\mu_{k}(r)$ and $\lambda_{k}(r)$ may be written

$$
\mu_{k}(r)=\sum_{\substack{d, r \mid r \\(a, r)_{k}=1}} \mu\left(\frac{r}{d}\right), \quad \lambda_{k}(r)=\sum_{a^{k} \mid r} \mu\left(\frac{r}{d^{k}}\right),
$$

In view of the factorability of $\mu_{k}(r)$ and $\lambda_{k}(r)$ it is sufficient to evaluate these functions for prime-power values of $r, r=p^{m}$ ( $p$ prime, $\left.m>0\right)$. In particular, it is easily deduced from (6.5) that

$$
\mu_{k}\left(p^{m}\right)=\left\{\begin{array}{rr}
-1 & (m=k) \\
0 & (m \neq k)
\end{array}\right.
$$

while for $k \geq 2$,

$$
\lambda_{k}\left(p^{m}\right)=\left\{\begin{aligned}
1 & (m \equiv 0(\bmod k)) \\
-1 & (m \equiv 1(\bmod k)) \\
0 & \text { (otherwise }) .
\end{aligned}\right.
$$

The functions $\mu_{k}(n)$ and $\lambda_{k}(n)$ were introduced by Gegenbauer [4]; for a further discussion we mention Hölder [6, §§6-7]. Note that $\lambda_{1}(r)=\mu_{0}(r)=\rho(r), \lambda_{0}(r)=\mu(r)$.

The corresponding inversion formulas are contained in the following relations (Theorem 3 ):

$$
\begin{aligned}
& f(r)=\sum_{d^{k} \mid r} g\left(\frac{r}{d}\right) \rightleftarrows g(r)=\sum_{d \mid r} f(d) \mu_{k}\left(\frac{r}{d}\right) ; \\
& f(r)=\sum_{\substack{d|r| r \\
(d-\text { rree })}} g\left(\frac{r}{d}\right) \rightleftarrows g(r)=\sum_{d \mid r} f(d) \lambda_{k}\left(\begin{array}{c}
r \\
d
\end{array}\right) .
\end{aligned}
$$

The case $k=1$ in (6.8) is the ordinary inversion theorem, while the case $k=2$ in (6.9) yields the formula,

$$
f(r)=\sum_{\substack{d \mid r \\
(\mu(d) \neq 0)}} g\left(\frac{r}{d}\right) \rightleftarrows g(r)=\sum_{d \mid r} f(d) \lambda\left(\begin{array}{l}
r \\
d
\end{array}\right),
$$

the summation on the left ranging over the primitive (square-free) divisors of $r$.

We now specialize the additive results of $\S 5$ to the particular sets $P, Q$ of this section. Placing $R_{k, s}(n, r)=A_{P}^{(s)}(n, r), S_{k, s}(n, r)=$ $A_{Q}^{(s)}(n, r)$, we observe that $R_{k, s}(n, r)$ represents the number of solutions of (1.3) such that $\left(x_{i}, r\right)_{k}=1$, while $S_{k, s}(n, r)$ represents the number of solutions of $(1.3)$ such that $\left(x_{i}, r\right)$ is a $k$ th power $(i=1, \cdots, s)$. In 
particular, one obtains from Theorem 12,

$$
\begin{aligned}
& R_{k, s}(n, r)=\frac{1}{r} \sum_{d \mid r}\left(g_{k}\left(\frac{r}{d}, r\right)\right)^{s} c(n, d), \\
& S_{k, s}(n, r)=\frac{1}{r} \sum_{a \mid r}\left(h_{k}\left(\frac{r}{d}, r\right)\right)^{s} c(n, d) .
\end{aligned}
$$

The case $k=1$ in (6.10) is Theorem 6 of [1], (also cf. [2, $\S 2]$ ), while the case $k=2$ in (6.11) is Theorem 3 of [3] in an equivalent form.

If one places $\theta_{P}(n, r)=\theta_{k}(n, r)$ and $\theta_{Q}(n, r)=\varepsilon_{k}(n, r)$, then $\theta_{k}(n, r)$ denote the number of integers $a(\bmod r)$ such that $(a, r)=1$ and $(n-a, r)_{k}=1$, while $\varepsilon_{k}(n, r)$ denotes the number of $a(\bmod r)$ such that $(a, r)=1$ and $(n-a, r)$ is a $k$ th power. We deduce then from Theorem 13,

$$
\begin{aligned}
& \theta_{k}(n, r)=\phi(r) \sum_{\substack{d \mid r \\
(l, n)=1}} \frac{\mu_{k}(d)}{\phi(d)}, \\
& \varepsilon_{k}(n, r)=\phi(r) \sum_{\substack{p \mid r \\
(l, n)=1}} \frac{\lambda_{k}(d)}{\phi(d)} .
\end{aligned}
$$

The case $k=1$ in (6.12) is [2, Corollary 21] while the case $k=2$ in (6.13) is [3, Corollary 38].

Finally, we investigate the conditions under which $\theta_{k}(n, r)$ and $\varepsilon_{k}(n, r)$ vanish. It is sufficient to consider these functions when $r$ and $n$ are powers of the same prime $p, r=p^{t}, n=p^{b}, t>0, t \geq b \geq 0$. A simple computation yields the following results. If $k \geq 1$, then

$$
\theta_{k}\left(p^{b}, p^{t}\right)= \begin{cases}p^{t-k}\left(p^{k}-p^{k-1}-1\right) & \text { if } b=0, t \geq k, \\ p^{t-1}(p-1) & \text { otherwise } .\end{cases}
$$

Suppose $a k<t \leq(a+1) k$ where $a$ is a (uniquely defined) non-negative integer. Then, if $k \leq 2$,

$$
\left(p^{k}-1\right) \varepsilon_{k}\left(p^{b}, p^{t}\right)=\left\{\begin{array}{l}
p^{t-1}(p-1)\left(p^{k}-1\right), \\
p^{t-k(a+1)}\left(p^{k-1}-1\right)+p^{k+t-1}(p-2)+p^{t-1} \\
p^{t+k-1}(p-2)+p^{t-a k-1}\left(p^{a k}-p+1\right),
\end{array}\right.
$$

according as (i) $b>0$, (ii) $b=0, t=(a+1) k$, or (iii) $b=0, t<(a+1) k$.

From these results it is easy to deduce that $\theta_{k}\left(p^{b}, p^{t}\right)=0$ if and only if $p=2, k=1, b=0$ and that $\varepsilon_{k}\left(p^{b}, p^{t}\right)=0$ if and only if $p=$ $2, t<k, b=0$. We are therefore led, on the basis of factorability considerations, to the following criterion in the general case.

Theorem 14. If $k \geq 1$, then $\theta_{k}(n, r)=0$ if and only if $k=1, r$ is even, and $n$ is odd.

If $k \geq 2$, then $\varepsilon_{k}(n, r)=0$ if and only if $r$ is of the form $2^{t} R$ where $R$ is odd, $0<t<k$, and $n$ is odd. 
The above result for $\theta_{k}(n, r)$ in case $k=1$ is due to Ramanathan [8, p. 68]. The result for $\varepsilon_{k}(n, r)$ in case $k=2$ was proved in [3, Corollary 38.1].

\section{REFERENCES}

1. Eckford Cohen, A class of arithmeical functions, Proc. Nat. Acad. Sci., 41 (1955), 939-944.

2. ...., Representation of even functions (mod $r$ ), I. Arithmetical identities, Duke Math. J. 25 (1958), 401-421.

3. - Representations of even funciions $(\bmod r)$, II. Cauchy products, Duke Math. J., to appear.

4. Leopold Gegenbauer, Zahlentheoretische Notiz, S.-B. Akad. Wiss. (Vienna), 97 (1889), IIa, $420-426$.

5. E. K. Haviland, An analogue of Euler's $\phi$-function, Duke Math. J., 11 (1944), 869-872.

6. O. Hölder, Über gewisse der Möbiusschen Funlttion $\mu(n)$ verwandie zahlentheoretische Funktionen, die Dirichletsche Multiplikation, und eine Verallgemeinerung der Umkehrungsformeln, Verh. Sachs. Akad. Wiss (Leipzig), 85 (1933), 3-28.

7. Paul. J. McCarthy, On a certain family of arithmetic functions, Amer. Math. Monthly, 65 (1958), 586-590.

8. K. G. Ramanathan, Some applications of Ramanujan's trigonometrical sum $C_{m}(n)$, Proc. Indian Acad. Sci. (A), 20 (1944), 62-69.

9. F. Rogel, Entwicklung einiger zahlentheoretischer Funktionen in unendliche, Reihen S.-B. Kgl. Böhmischen Ges. Wiss. 30 (1900).

UNIVERSity OF TENNESSEE 



\section{PACIFIC JOURNAL OF MATHEMATICS}

\section{EDITORS}

\section{David Gilbarg}

Stanford University

Stanford, California

R. A. Beaumont

University of Washington

Seattle 5 , Washington
A. L. Whiteman

University of Southern California

Los Angeles 7, California

L. J. PAIGE

University of California

Los Angeles 24, California

\section{ASSOCIATE EDITORS}
E. F. BECKENBACH
C. E. BURGESS
E. HEWITT
A. HORN

\author{
V. GANAPATHY IYER \\ R. D. JAMES \\ M. S. KNEBELMAN \\ L. NACHBIN
}
I. NIVEN
E. G. STRAUS
T. G. OSTROM
H. L. ROYDEN
G. SZEKERES
M. M. SCHIFFER
F. WOLF
K. YOSIDA

\section{SUPPORTING INSTITUTIONS}

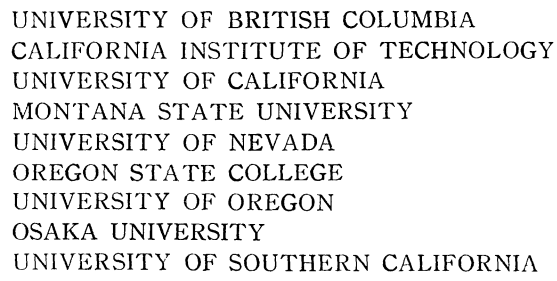

UNIVERSITY OF BRITISH COLUMBIA CALIFORNIA INSTITUTE OF TECHNOLOGY UNIVERSITY OF CALIFORNIA MONTANA STATE UNIVERSITY UNIVERSITY OF NEVADA OREGON STATE COLLEGE UNIVERSITY OF OREGON OSAKA UNIVERSITY UNIVERSITY OF SOUTHERN CALIFORNIA

STANFORD UNIVERSITY

UNIVERSITY OF TOKYO

UNIVERSITY OF UTAH

WASHINGTON STATE COLLEGE

UNIVERSITY OF WASHINGTON

AMERICAN MATHEMATICAL SOCIETY CALIFORNIA RESEARCH CORPORATION HUGHES AIRCRAFT COMPANY SPACE TECHNOLOGY LABORATORIES

Printed in Japan by Kokusai Bunken Insatsusha (International Academic Printing Co., Ltd.), Tokyo, Japan 


\section{Pacific Journal of Mathematics}

\section{Vol. 9, No. 1 \\ May, 1959}

Julius Rubin Blum and Murray Rosenblatt, On the structure of infinitely

divisible distributions . ............................. 1

Robert Geroge Buschman, Asymptotic expressions for

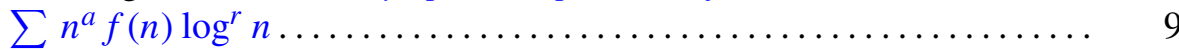

Eckford Cohen, A class of residue systems $(\bmod r)$ and related arithmetical

functions. I. A generalization of Möbius inversion .............. 13

Paul F. Conrad, Non-abelian ordered groups ................... 25

Richard Henry Crowell, On the van Kampen theorem............... 43

Irving Leonard Glicksberg, Convolution semigroups of measures ........ 51

Seymour Goldberg, Linear operators and their conjugates ............ 69

Olof Hanner, Mean play of sums of positional games .............. 81

Erhard Heinz, On one-to-one harmonic mappings ................ 101

John Rolfe Isbell, On finite-dimensional uniform spaces . . ........... 107

Erwin Kreyszig and John Todd, On the radius of univalence of the function

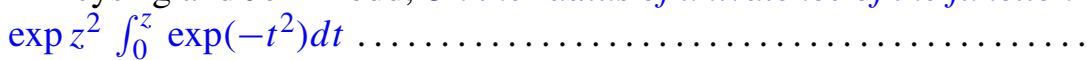

Roger Conant Lyndon, An interpolation theorem in the predicate

calculus......................................... 129

Roger Conant Lyndon, Properties preserved under homomorphism ........ 143

Roger Conant Lyndon, Properties preserved in subdirect products ....... 155

Robert Osserman, A lemma on analytic curves ................ 165

R. S. Phillips, On a theorem due to Sz.-Nagy..................... 169

Richard Scott Pierce, A generalization of atomic Boolean algebras ....... 175

J. B. Roberts, Analytic continuation of meromorphic functions in valued fields................................. 183

Walter Rudin, Idempotent measures on Abelian groups ................ 195

M. Schiffer, Fredholm eigen values of multiply-connected domains ........ 211

V. N. Singh, A note on the computation of Alder's polynomials ......... 271

Maurice Sion, On integration of 1-forms ...................... 277

Elbert A. Walker, Subdirect sums and infinite Abelian groups........... 287

John W. Woll, Homogeneous stochastic processes . .................. 293 